\title{
Características das instalações e equipamentos para a prática de atividade física em escolas da zona rural de Pelotas, Rio Grande do Sul
}

\author{
Facilities and equipments for physical activity practice in rural schools of Pelotas, \\ Rio Grande do Sul
}

\section{AUTORES \\ Werner de Andrade Müller ${ }^{1}$ (D) \\ Gabriele Radünz Krüger ${ }^{1}$ \\ Marlos Rodrigues Domingues ${ }^{1,2}$ (D) \\ 1 Programa de Pós-graduação em Educação Física, Universidade Federal de Pelotas, Pelotas, Rio Grande do Sul, Brasil. \\ 2 Escola Superior de Educação Física, Universidade Federal de Pelotas, Pelotas, Rio Grande do Sul, Brasil.}

\section{CONTATO}

\section{Werner de Andrade Müller}

werneramuller@gmail.com

Escola Superior de Educação Física,

Universidade Federal de Pelotas. Rua Luís

de Camões, 625, Três Vendas, Pelotas, Rio

Grande do Sul. Brasil.

CEP: 96055-630.

DOI

$10.12820 /$ rbafs. $24 \mathrm{e} 0077$

\begin{abstract}
RESUMO
O objetivo do estudo foi descrever as instalações e equipamentos para atividade física nas escolas da zona rural de Pelotas, Rio Grande do Sul. Trata-se de um estudo transversal, de base escolar, realizado no ano de 2014, em todas as 26 escolas localizadas na zona rural da cidade de Pelotas. Foi utilizado um instrumento com questões relacionadas às políticas de Educação Física e avaliação das estruturas e materiais para a prática de atividade física, elaborado a partir do Physical Activity Resource Assessment (PARA), adaptado para o ambiente escolar. Observou-se que o professor e a disciplina de Educação Física estiveram presentes em todas as escolas. Mesmo com a maior frequência de estruturas como quadras esportivas $(34,6 \%$ a $65,4 \%)$, pracinha/parquinhos $(76,9 \%)$ e materiais como bolas $(50,0 \%$ a $84,6 \%)$, cones $(80,8 \%)$, cordas $(88,5 \%)$, colchonetes $(80,8 \%)$ e $\operatorname{arcos}(76,9 \%)$, as condições dos atributos foram regulares $(7,7 \%$ a $46,2 \%)$ e ruins $(11,5 \%$ a $50,0 \%)$. Poucas instituições ofereceram instalações e equipamentos em condições adequadas para a prática de atividade física. A ausência de ginásios, quadras cobertas e materiais em melhores condições chama a atenção para a necessidade de investimentos e intervenções nesses espaços.
\end{abstract}

Palavras-chave: Planejamento ambiental; População rural; Atividade motora; Políticas públicas de saúde; Instituições acadêmicas.

\begin{abstract}
The aim of the study was to describe the facilities and equipment for physical activity practice in rural area schools of Pelotas, Rio Grande do Sul. The design was cross-sectional, school-based, carried out in all the 26 schools located in rural areas of Pelotas Southern, Brazil. We used a survey tool to assess the policies for Physical Education and evaluate the facilities and equipment/materials for physical activity. The instrument was based on the Physical Activity Resource Assessment (PARA), adapted for the school environment. We observed that curricular Physical Education and specific teachers were present in all schools. Despite the high number of facilities, such as courts and fields (34.6\% to 65.4\%), playgrounds (76.9\%) and materials such as balls (50.0\% to 84.6\%), hoops (88.5\%), exercise cones (80.8\%) and mats (76.9\%), the condition of the facilities and apparatus/equipment were fair (7.7\% to $46.2 \%$ ) or poor (11.5\% to 50.0\%). Few institutions have provided suitable facilities and equipment for physical activity. The absence of gymnasiums, covered courts and materials in better conditions draws attention to the need for investments and interventions in these spaces.
\end{abstract}

Keywords: Environment design; Rural population; Motor activity; Public health policy; Schools.

\section{(c) BY-NC-SA}

Este obra está licenciado com uma Licença

Creative Commons Atribuição-NãoComercial-

CompartilhaIgual 4.0 Internacional.

\section{Introdução}

A prática de atividade física $(\mathrm{AF})$ na adolescência está associada a benefícios tanto de curto quanto longo prazo que incluem a melhora da saúde física, mental e cognitiva ${ }^{1}$. Dados mostram que apenas $1 / 4$ dos ado- lescentes atingem as recomendações de AF em nível global $^{2}$ e menos da metade no Brasil ${ }^{3}$. Em populações rurais essa prevalência se mantém parecida ${ }^{4,5}$.

O comportamento fisicamente ativo pode ser afetado por variáveis de diferentes níveis, sendo um deles 
o ambiental ${ }^{6}$. Assim, os ambientes de casa, bairro e da escola podem ser determinantes de hábitos saudáveis ${ }^{7}$. $\mathrm{Na}$ escola, a disciplina de Educação Física é considerada uma intervenção efetiva para a promoção de saúde ${ }^{8}$, porém, mesmo como uma importante fonte de $\mathrm{AF}^{9}$, o tempo de envolvimento dos alunos em atividades que contribuem para a melhoria da saúde durante as aulas de Educação Física equivale a apenas um terço da aula ${ }^{10}$.

As características do ambiente escolar, como instalações, equipamentos, oferta e desenvolvimento de atividades, oferta de recreio e atividades supervisionadas podem ser potenciais influenciadores nos níveis de AF e participação em atividades dos escolares ${ }^{11-13}$. Diversos atributos, mesmo que de forma isolada e em diferentes contextos, acerca de melhorias nos espaços, políticas de promoção de saúde ou percepções positivas do ambiente, parecem se relacionar com a maior prática de AF de crianças e adolescentes na escola ${ }^{14}$.

Sallis et al. ${ }^{15} \mathrm{em}$ um estudo pioneiro realizado nos Estados Unidos no início dos anos 2000, sobre a relação do ambiente da escola com o comportamento de AF, constataram que as características ambientais explicaram $42 \%$ e 59\%, respectivamente, da proporção de meninas e meninos que eram fisicamente ativos, indicando que ambientes com melhorias estimularam um comportamento ativo nos estudantes. Uma série de outras pesquisas foram desenvolvidas de forma a consolidar os achados corroborados ${ }^{16}$ e da mesma forma que compreender a relação do ambiente com o comportamento de AF tornou-se importante, avaliar o ambiente escolar torna-se fundamental para se intervir em ambientes e comportamentos mais ativos.

Em pesquisa realizada no Recife, Pernambuco, que investigou características estruturais de escolas regulares e de um programa de educação integral, verificou que a maioria das escolas possuía quadra de esportes e materiais para Educação Física. No entanto, a maior parte das instituições apresentaram espaços, proteção e equipamentos de menor qualidade ${ }^{17}$. Em Pelotas, Rio Grande do Sul, 10,1\% das 99 escolas com ensino fundamental da zona urbana apresentaram ginásios e estruturas como quadras esportivas. Salas internas e áreas verdes foram encontradas em maior quantidade e qualidade nas escolas particulares ${ }^{18}$. Nesse contexto, avaliar as características do ambiente escolar para a promoção de AF se torna importante para planejar intervenções e políticas públicas.

As escolas rurais de educação básica apresentam características próprias. De acordo com o Instituto
Nacional de Estudos e Pesquisas Educacionais do Ministério da Educação, a estrutura física e os recursos disponíveis são precários em comparação às escolas urbanas, com condições desvantajosas de trabalho e para o funcionamento das mesmas. Os estabelecimentos são, em sua maioria, de pequeno porte, com oferta de ensino fundamental e atendem até 50 alunos ${ }^{19}$.

Considerando a escassez de estudos sobre o ambiente escolar em escolas rurais, o presente estudo tem como objetivo descrever as instalações e equipamentos para a prática de AF de escolas localizadas na zona rural de Pelotas, Rio Grande do Sul.

\section{Métodos}

Trata-se de um estudo observacional transversal, do tipo censo, realizado em escolas da zona rural de Pelotas, Rio Grande do Sul. O estudo foi aprovado pelo Comitê de Ética em Pesquisa com Seres Humanos da Faculdade de Medicina da Universidade Federal de Pelotas (CAAE: 3883814.1.0000.5317). Todos os participantes assinaram o Termo de Consentimento Livre e Esclarecido.

A coleta de dados foi realizada no segundo semestre de 2014, a partir da avaliação do ambiente escolar e entrevistas com professores e gestores das escolas. As entrevistas priorizaram a participação de um membro da equipe diretiva junto com o professor de Educação Física e na falta do gestor no dia da coleta de dados, apenas o professor era entrevistado.

Foram incluídas no estudo todas as escolas localizadas na zona rural de Pelotas. De acordo com a Secretaria Municipal de Educação e Desportos e a $5^{\text {a }}$ Coordenadoria Regional de Educação do Rio Grande do Sul, 28 escolas - 5 estaduais e 23 municipais - estão distribuídas nos oito distritos rurais do município. Foram excluídas as escolas que não estivessem em funcionamento no momento da realização do estudo.

O trabalho de campo foi conduzido por um único avaliador e seguiu critérios pré-estabelecidos por um protocolo técnico operacional. Foi realizado um estudo piloto anterior à coleta de dados, em uma escola não pertencente a zona rural, mas com características similares, para testar a aplicação do instrumento e aprimorar a logística. Além disso, foi feito o registro fotográfico dos espaços verificados que garantiu um controle de qualidade da coleta de dados, visto que, após a aplicação do instrumento de pesquisa, os dados coletados eram conferidos com as fotos registradas.

A avaliação do ambiente escolar foi realizada a partir da aplicação de um instrumento composto por dois 
blocos, com questões organizacionais e de instalações e equipamentos para a prática de $\mathrm{AF}$, baseado em estudos realizados no âmbito escolar na cidade de Pelotas ${ }^{20,21} \mathrm{e}$ no instrumento internacional de avaliação de estruturas para a prática de AF Physical Activity Resource Assessment (PARA)22. O PARA foi adaptado para o ambiente da escola de forma a contemplar todos os espaços de uso para a prática de AF e lazer que podem ser encontrados nos ambientes escolares para a avaliação das estruturas, bem como equipamentos de uso esportivo, de lazer e de Educação Física para os materiais.

O primeiro bloco com informações organizacionais das escolas foi feito em forma de entrevista com um representante da equipe diretiva ou professor de Educação Física, com questões referentes ao: tipo de escola enquanto rede de ensino municipal ou estadual; etapas de ensino em ensino fundamental incompleto, ensino fundamental completo, ensino fundamental e médio ou ensino médio; turnos de funcionamento em manhã, tarde e noite; quantitativo de alunos, professores e professores de Educação Física; frequência semanal de aulas de Educação Física por etapa de ensino; oferta de recreio e de atividades extracurriculares.

O segundo bloco foi baseado na checagem da quantidade e condições das instalações e materiais presentes para a prática de AF na escola, além de aspectos de limpeza, estética e segurança do ambiente. As estruturas físicas foram identificadas a partir da presença de quadras, campos e espaços para a prática de esportes e áreas de lazer e classificadas de acordo com uma escala de 0 a 3, sendo "0" para a ausência da estrutura, "1" para estruturas de qualidade ruim, "2" de qualidade regular e " 3 " de qualidade boa, de acordo com atributos específicos para cada item.

Os materiais foram classificados a partir da quantidade e qualidade, seguindo definições específicas para cada item verificado. Foram classificados em " 0 " na ausência do material; "1" para qualidade ruim: em que pelo menos $90 \%$ apresentassem condições ruins ou de desgaste; "2" para qualidade regular: de 30\% a 89\% dos materiais em condições ruins ou de desgaste; e " 3 " para qualidade boa: com até $10 \%$ dos materiais em condições ruins ou de desgaste. Os materiais verificados foram: bolas de diversas modalidades e tipos, materiais de atletismo, cones, cordas, colchonetes, arcos, halteres, redes e demais equipamentos encontrados na escola.

Para aspectos de estética, limpeza e segurança foi verificada a presença de evidências que pudessem comprometer a utilização do espaço (lixo, vidro quebrado, dejeto de animais, animais soltos, sinais de vandalismo, pichações, uso de álcool e tabaco e mato ou grama alta). Tais evidências foram classificadas, individualmente, de 0 a 2 , sendo "0" para a inexistência da mesma; "1" para existência em menor quantidade, com pelo menos um indício da evidência; e "2" para existência em maior quantidade, contabilizada a partir de dois indícios encontrados. Desta forma, quanto maior o indício de evidências, maior a deficiência do local e pior qualidade para o uso.

A elaboração dos escores de qualidade de estruturas e de materiais foi realizada por meio da soma dos itens verificados, multiplicada pela qualidade dos mesmos. A fim de diminuir a amplitude dos escores, o produto da multiplicação foi dividido em quintis e atribuído pontuações de 1 a 5 , sendo 1 de menor qualidade e 5 de maior qualidade. No quintil mais baixo foi conferido 1 ponto e, de modo sucessivo nos demais, 5 pontos no quintil mais alto, resultando em um escore final que variou de 0 a 25 pontos em amplitude.

O escore de materiais, seguindo as mesmas definições, possuiu a amplitude de 0 a 50, uma vez que foi elaborado a partir da soma do escore de bolas com o escore de outros materiais (arcos, cones, colchonetes e entre outros). Utilizou-se a soma de dois escores de materiais em virtude de o número de outros materiais ser superior comparado ao número de bolas. Não fazendo sentido realizar uma soma simples de quatro cones com quatro bolas, em mesmas condições, por exemplo, acreditando que os materiais exemplificados possuem importâncias distintas no contexto escolar. Em ambos os escores - de estruturas e de materiais -, os valores mais altos e mais baixos refletiram, respectivamente, maior e menor qualidade.

As informações coletadas foram duplamente digitadas no programa EpiData 3.1 e transferidos para o programa Stata IC 12.0. A distribuição dos dados foi descrita através da frequência absoluta e relativa. Para a análise da qualidade das estruturas e materiais, de acordo com o tipo de escola, foi utilizado média e desvio-padrão.

\section{Resultados}

Foram localizadas 26 escolas da zona rural de Pelotas. Dois estabelecimentos de ensino foram desativados desde o último levantamento dos órgãos de educação do município. A maioria das instituições pesquisadas eram municipais $(80,1 \%)$, possuía até 150 alunos $(65,4 \%)$ e funcionava nos turnos de manhã e tarde $(69,3 \%)$. Todas as escolas contavam com professores formados, aulas de Educação Física e oferta de recreio. 
Atividades extracurriculares foram ofertadas em 65,4\% das escolas (Tabela 1).

A atividade extracurricular mais frequente foi torneio esportivo interno, realizada em $53,6 \%$ das instituições. Futsal, atletismo, xadrez e voleibol estiveram presentes em 10,0\% das escolas (informações não apresentadas nas tabelas).

A proporção de escolas que possuíam determinadas estruturas para a prática de AF está disposta na Tabela 2. As instalações mais presentes foram pracinha/ parquinho $(76,9 \%)$, quadras externas $(65,4 \%$ de voleibol, $46,1 \%$ de futsal, $38,5 \%$ de handebol e $34,6 \%$ de basquetebol) e campo de futebol (42,3\%). A maioria das escolas (de 30,8\% a 46,2\%) contou com estruturas em condições ruins e regulares de preservação. Entre as escolas que apresentaram quadras externas, apenas sete $(26,9 \%)$ eram cobertas. Estruturas como ginásio, quadras internas, salas de dança e ginástica e vestiários não foram encontradas em nenhuma escola.

Em relação aos materiais de Educação Física, na maior parte das escolas os materiais mais comuns foram: cordas $(88,5 \%)$, rede de voleibol $(84,6 \%)$, bolas de basquetebol $(84,86)$, de voleibol $(84,6 \%)$, futebol $(80,8 \%)$, colchonete $(80,8 \%)$, cones $(80,5 \%)$, arcos $(76,9 \%)$, bolas de handebol $(69,2 \%)$ e futsal $(61,5 \%)$ - Tabela 3. A qualidade dos materiais variou, principalmente, de ruim para regular, sendo que cone foi o item que apresentou melhor condição de uso $(50,0 \%$ e $30,8 \%$ qualidade regular a boa, respectivamente) e bolas de futebol a pior condição de conservação (50,0\% em condição ruim). Outros materiais foram encontrados em menor número e também apresentaram qualidade ruim a regular, sendo: bola de tênis, raquete, bloco de partida, bússola e tabuleiro de xadrez (dados não apresentados).

Referente às condições de estética, limpeza e segurança dos espaços escolares, a Tabela 4 apresenta que mais da metade das escolas não apresentou vidro quebrado (92,3\%), resquícios de uso de álcool $(92,3 \%)$ e tabaco $(84,6 \%)$, dejeto de animais $(80,8 \%)$, sinais de vandalismo $(61,5 \%)$ e pichações $(65,4 \%)$. Itens como lixo espalhado, animais soltos e mato ou grama alta foram observados em $73,1 \%, 34,6 \%$ e $34,6 \%$, respectivamente, nos ambientes verificados.

Quando analisado o escore para a qualidade das estruturas de acordo com as características das escolas, se evidenciou que as instituições de ensino básico completo, com maior número de alunos e com maior número de professores de Educação Física, obtiveram maior média absoluta de pontos no escore de qualidade
Tabela 1 - Características das escolas localizadas na zona rural de Pelotas, Rio Grande do Sul, 2014.

\begin{tabular}{|c|c|c|}
\hline Características & $\mathrm{n}=26$ & $\%$ \\
\hline \multicolumn{3}{|l|}{ Tipo de escola } \\
\hline Estadual & 5 & 19,2 \\
\hline Municipal & 21 & 80,8 \\
\hline \multicolumn{3}{|l|}{ Etapa de ensino ${ }^{a}$} \\
\hline Fundamental incompleto & 9 & 34,6 \\
\hline Fundamental completo & 14 & 53,9 \\
\hline Fundamental e médio & 1 & 3,8 \\
\hline Médio & 2 & 7,7 \\
\hline \multicolumn{3}{|l|}{ Turno das aulas } \\
\hline Manhã & 1 & 3,9 \\
\hline Manhã e tarde & 17 & 65,2 \\
\hline Tarde e noite & 1 & 3,9 \\
\hline Noite & 1 & 3,9 \\
\hline Manhã, tarde e noite & 6 & 23,1 \\
\hline \multicolumn{3}{|l|}{ Número de alunos } \\
\hline Até 49 & 7 & 26,9 \\
\hline 50 a 149 & 10 & 38,5 \\
\hline 150 a 299 & 6 & 23,1 \\
\hline 300 ou mais & 3 & 11,5 \\
\hline \multicolumn{3}{|l|}{ Número de professores } \\
\hline Até 9 & 10 & 38,5 \\
\hline 10 a 19 & 5 & 19,2 \\
\hline 20 a 29 & 5 & 19,2 \\
\hline 30 ou mais & 6 & 23,1 \\
\hline \multicolumn{3}{|c|}{ Número de professores de Educação Física } \\
\hline $\mathrm{Um}$ & 16 & 61,6 \\
\hline Dois & 7 & 26,9 \\
\hline Três & 3 & 11,5 \\
\hline \multicolumn{3}{|c|}{$\begin{array}{l}\text { Número de aulas de Educação Física até o } \\
5^{\circ} \text { ano }(\mathrm{n}=24)^{\mathrm{b}}\end{array}$} \\
\hline Nenhuma & 0 & 0,0 \\
\hline Uma a duas & 20 & 83,3 \\
\hline Três & 4 & 16,7 \\
\hline \multicolumn{3}{|c|}{$\begin{array}{l}\text { Número de aulas de Educação Física do } 5^{\circ} \\
\text { ao } 9^{\mathrm{o}} \text { ano }(\mathrm{n}=15)^{\mathrm{b}}\end{array}$} \\
\hline Nenhuma & 0 & 0,0 \\
\hline Uma a duas & 8 & 53,3 \\
\hline Três & 7 & 46,7 \\
\hline \multicolumn{3}{|c|}{$\begin{array}{l}\text { Número de aulas de Educação Física no } \\
\text { ensino médio }(n=03)^{b}\end{array}$} \\
\hline Nenhuma & 0 & 0,0 \\
\hline Uma a duas & 3 & 100,0 \\
\hline Três & 0 & 0,0 \\
\hline \multicolumn{3}{|l|}{ Oferta de recreio } \\
\hline $1^{\mathrm{o}}$ ao $5^{\mathrm{o}}$ ano & 24 & 100,0 \\
\hline $5^{\circ}$ ao $9^{\circ}$ ano & 15 & 100,0 \\
\hline Ensino médio & 3 & 100,0 \\
\hline \multicolumn{3}{|c|}{ Oferta de atividades extracurriculares } \\
\hline Sim & 17 & 65,4 \\
\hline Não & 9 & 34,6 \\
\hline
\end{tabular}

$\mathrm{a}=$ na etapa de ensino fundamental está compreendida também a pré-escola da educação infantil; $\mathrm{b}$ = número de escolas de acordo com a oferta de ensino. 
Tabela 2 - Proporção de escolas da zona rural de Pelotas, Rio Grande do Sul que possuem instalações para a prática de atividade, de acordo com a condição dos espaços, 2014.

\begin{tabular}{|c|c|c|c|c|c|c|c|c|}
\hline \multirow{3}{*}{ Estrutura } & \multicolumn{6}{|c|}{ Qualidade } & \multirow{2}{*}{\multicolumn{2}{|c|}{$\frac{\text { Quantidade }}{\text { Total }}$}} \\
\hline & \multicolumn{2}{|c|}{ Ruim } & \multicolumn{2}{|c|}{ Regular } & \multicolumn{2}{|c|}{ Boa } & & \\
\hline & $\mathrm{n}$ & $\%$ & $\mathrm{n}$ & $\%$ & $\mathrm{n}$ & $\%$ & $\mathrm{n}$ & $\%$ \\
\hline Pracinha/parquinho & 7 & 26,9 & 12 & 46,2 & 1 & 3,9 & 20 & 76,9 \\
\hline Quadra de voleibol externa & 9 & 34,6 & 6 & 23,1 & 2 & 7,7 & 17 & 65,4 \\
\hline Quadra de futsal externa & 3 & 11,5 & 6 & 23,1 & 3 & 11,5 & 12 & 46,1 \\
\hline Campo de futebol & 8 & 30,8 & 3 & 11,5 & 0 & 0,0 & 11 & 42,3 \\
\hline Quadra de handebol externa & 2 & 7,7 & 6 & 23,1 & 2 & 7,7 & 10 & 38,5 \\
\hline Quadra de basquetebol externa & 5 & 19,2 & 2 & 7,7 & 2 & 7,7 & 9 & 34,6 \\
\hline Pista de salto & 2 & 7,7 & 1 & 3,9 & 0 & 0,0 & 3 & 11,5 \\
\hline Pista de corrida & 2 & 7,7 & 0 & 0,0 & 0 & 0,0 & 2 & 7,7 \\
\hline Sala de recreação & 0 & 0,0 & 1 & 3,9 & 0 & 0,0 & 1 & 3,9 \\
\hline
\end{tabular}

Tabela 3 - Proporção de escolas da zona rural de Pelotas, Rio Grande do Sul que possuem materiais para a prática de atividade física, de acordo com a condição dos materiais, 2014.

\begin{tabular}{|c|c|c|c|c|c|c|c|c|}
\hline \multirow{3}{*}{ Materiais } & \multicolumn{6}{|c|}{ Qualidade } & \multirow{2}{*}{\multicolumn{2}{|c|}{$\begin{array}{c}\text { Quantidade } \\
\text { Total }\end{array}$}} \\
\hline & \multicolumn{2}{|c|}{ Ruim } & \multicolumn{2}{|c|}{ Regular } & \multicolumn{2}{|c|}{ Boa } & & \\
\hline & $\mathrm{n}$ & $\%$ & $\mathrm{n}$ & $\%$ & $\mathrm{n}$ & $\%$ & $\mathrm{n}$ & $\%$ \\
\hline Cordas & 11 & 42,3 & 8 & 30,8 & 4 & 15,4 & 23 & 88,5 \\
\hline Rede de voleibol & 10 & 38,5 & 10 & 38,5 & 2 & 7,7 & 22 & 84,6 \\
\hline Bola de voleibol & 5 & 19,2 & 13 & 50,0 & 4 & 15,4 & 22 & 84,6 \\
\hline Bola de basquetebol & 3 & 11,5 & 14 & 53,9 & 5 & 19,2 & 22 & 84,6 \\
\hline Bola de futebol & 13 & 50,0 & 6 & 23,1 & 2 & 7,7 & 21 & 80,8 \\
\hline Colchonete & 9 & 34,6 & 8 & 30,8 & 4 & 15,4 & 21 & 80,8 \\
\hline Cones & 0 & 0,0 & 13 & 50,0 & 8 & 30,8 & 21 & 80,5 \\
\hline $\operatorname{Arcos}$ & 10 & 38,5 & 7 & 26,9 & 3 & 11,5 & 20 & 76,9 \\
\hline Bola de handebol & 4 & 15,4 & 12 & 46,2 & 2 & 7,7 & 18 & 69,2 \\
\hline Bola de futsal & 4 & 15,4 & 7 & 26,9 & 5 & 19,2 & 16 & 61,5 \\
\hline Bola de borracha & 5 & 19,2 & 5 & 19,2 & 3 & 11,5 & 13 & 50,0 \\
\hline Medicine ball & 1 & 3,9 & 3 & 11,5 & 3 & 11,5 & 7 & 26,9 \\
\hline Bastão atletismo & 1 & 3,9 & 1 & 3,9 & 4 & 15,4 & 6 & 23,1 \\
\hline Peso atletismo & 1 & 3,9 & 4 & 15,4 & 1 & 3,9 & 6 & 23,1 \\
\hline Disco atletismo & 1 & 3,9 & 1 & 3,9 & 2 & 7,7 & 4 & 15,4 \\
\hline Colchão de salto & 0 & 0,0 & 0 & 0,0 & 2 & 7,7 & 2 & 7,7 \\
\hline Halteres & 0 & 0,0 & 0 & 0,0 & 1 & 3,9 & 1 & 3,9 \\
\hline
\end{tabular}

Tabela 4 - Características de limpeza, estética e segurança das escolas da zona rural de Pelotas, Rio Grande do Sul, 2014.

\begin{tabular}{|c|c|c|c|c|c|c|}
\hline \multirow[t]{2}{*}{ Itens } & \multicolumn{2}{|c|}{ Inexistência } & \multicolumn{2}{|c|}{$\begin{array}{c}\text { Existência em menor } \\
\text { quantidade }\end{array}$} & \multicolumn{2}{|c|}{$\begin{array}{l}\text { Existência em maior } \\
\text { quantidade }\end{array}$} \\
\hline & $\mathrm{n}$ & $\%$ & $\mathrm{n}$ & $\%$ & $\mathrm{n}$ & $\%$ \\
\hline Vidro quebrado & 24 & 92,3 & 2 & 7,7 & 0 & 0,0 \\
\hline Dejeto de animais & 21 & 80,8 & 4 & 15,4 & 1 & 3,8 \\
\hline Animal solto & 13 & 50,0 & 9 & 34,6 & 4 & 15,4 \\
\hline Lixo espalhado & 1 & 3,9 & 6 & 23,1 & 19 & 73,0 \\
\hline Pichação & 17 & 65,4 & 3 & 11,5 & 6 & 23,1 \\
\hline Sinal de vandalismo & 16 & 61,5 & 6 & 23,1 & 4 & 15,4 \\
\hline Mato ou grama alta & 8 & 30,8 & 9 & 34,6 & 9 & 34,6 \\
\hline Evidência do uso de álcool & 24 & 92,3 & 2 & 7,7 & 0 & 0,0 \\
\hline Evidência do uso de tabaco & 22 & 84,6 & 0 & 0,0 & 4 & 15,4 \\
\hline
\end{tabular}


Tabela 5 - Média de pontuação da qualidade das estruturas e dos materiais para a prática de atividade física, de acordo com as características das escolas da zona rural de Pelotas, Rio Grande de Sul, 2014.

\begin{tabular}{|c|c|c|c|c|}
\hline \multirow[t]{2}{*}{ Características } & \multicolumn{2}{|c|}{$\begin{array}{l}\text { Escore estruturas } \\
(0-25 \text { pontos })\end{array}$} & \multicolumn{2}{|c|}{$\begin{array}{l}\text { Escore materiais } \\
(0-50 \text { pontos })\end{array}$} \\
\hline & Média & Desvio padrão & Média & Desvio padrão \\
\hline \multicolumn{5}{|l|}{ Tipo de Escola } \\
\hline Municipal & 15,47 & 7,22 & 33,57 & 12,05 \\
\hline Estadual & 13,00 & 5,70 & 19,00 & 12,44 \\
\hline \multicolumn{5}{|l|}{ Número de alunos } \\
\hline Até 49 & 10,00 & 5,00 & 25,71 & 9,32 \\
\hline $50-199$ & 11,50 & 4,11 & 29,00 & 16,63 \\
\hline $150-299$ & 22,50 & 2,73 & 40,83 & 8,01 \\
\hline Mais de 300 & 23,33 & 2,86 & 28,33 & 10,40 \\
\hline \multicolumn{5}{|l|}{ Etapa de ensino } \\
\hline Fundamental incompleto & 10,55 & 4,63 & 26,11 & 11,93 \\
\hline Fundamental completo & 18,57 & 6,33 & 35,35 & 12,77 \\
\hline Fundamental e médio & 20,00 & - & 20,00 & - \\
\hline Médio & 7,50 & 3,53 & 25,00 & 21,21 \\
\hline \multicolumn{5}{|l|}{ Professores de Educação Física } \\
\hline $\mathrm{Um}$ & 11,56 & 5,69 & 27,50 & 14,02 \\
\hline Dois & 18,57 & 4,75 & 36,42 & 11,80 \\
\hline Três & 25,00 & 0,00 & 35,00 & 8,66 \\
\hline \multicolumn{5}{|l|}{ Atividades Extracurriculares } \\
\hline Não & 12,77 & 4,40 & 28,33 & 15,61 \\
\hline $\operatorname{Sim}$ & 16,17 & 7,81 & 32,05 & 12,12 \\
\hline Total & 15,00 & 6,92 & 30,76 & 13,24 \\
\hline
\end{tabular}

das estruturas. Em relação ao escore da qualidade dos materiais de Educação Física, a média de pontos foi semelhante no que tange às características das escolas, obtendo maiores médias absolutas de escores somente as escolas municipais, com número de alunos entre 150 e 299 e com ensino fundamental completo (Tabela 5).

\section{Discussão}

Todas as escolas contaram com aulas e a presença do professor de Educação Física e as estruturas mais presentes foram quadras esportivas e pracinha/parquinhos. Materiais como bolas, arcos, cordas e colchonetes prevaleceram, porém em qualidade ruim.

O professor de Educação Física esteve presente em todas as escolas. Achados que corroboram com estudo realizado em 99 escolas da zona urbana de Pelotas, Rio Grande do Sul, em que seis escolas não contaram com a presença do professor $^{18}$ e com outras pesquisas desenvolvidas em capitais do país ${ }^{17,23}$. Constatou-se que em todas as escolas a disciplina de Educação Física esteve presente, sendo ministrada de uma a duas vezes por semana, diferente do estudo conduzido em Recife, Pernambuco, no qual 1/3 das escolas regulares da rede estadual oferecia duas aulas semanais ${ }^{17}$. Em nível nacional, dados da Pesquisa Nacional de Saúde do Escolar - PeNSE -, mostraram que apenas 37,3\% dos alunos matriculados no $9^{\circ}$ ano das escolas das capitais do país tinham duas aulas de Educação Física por semana ${ }^{24}$. Mesmo com a presença do professor de Educação Física em todas as escolas, em duas delas o professor não era responsável por lecionar nas séries iniciais do ensino fundamental. Situação amparada pela Lei LDB - 9394/1996 que, apesar da disciplina ser integrada à proposta da escola e componente curricular obrigatório da educação básica, não existe especificação que indique qual o profissional deve atuar nessa etapa de ensino ${ }^{25}$.

Referente à estrutura física da escola, observou-se que todas as instalações presentes se caracterizaram como externas, sendo: campos de futebol, quadras esportivas, pracinha/parquinhos e pistas de corrida e salto em distância. Apenas sete escolas contaram com cobertura nas quadras e em uma escola foi constatado haver 
uma sala interna para atividades relacionadas à Educação Física. Este fato é preocupante, visto que ausência de instalações específicas pode impedir o desenvolvimento de atividades curriculares e modificar o andamento das aulas, havendo o comprometimento do trabalho em função da meteorologia, como exposição ao sol, dias de chuva e segurança de alunos e professores ${ }^{26}$. Cabe ressaltar que as estruturas encontradas não estão adequadas ao clima da região da área de estudo, onde existe grande sazonalidade climática, típica das estações do ano no sul do país, com altos índices pluviométricos, temperaturas baixas no inverno e elevadas no verão.

No estudo realizado por Knuth el at. ${ }^{18}$, na zona urbana de Pelotas, apenas 4,8\% das escolas públicas possuíam ginásio, enquanto que $86,9 \%$ contavam com quadras externas, de um total de 84 escolas públicas com ensino fundamental no município. Tais achados corroboram com o baixo número de escolas com instalações abrigadas das variações do tempo e temperatura encontrados no presente estudo. No entanto, esses dados diferem da literatura internacional ${ }^{12}$, em que as escolas apresentaram elevado número de ginásios, instalações de chuveiros e vestiários. Além disso, a literatura internacional mostra que a maior parte das escolas pesquisadas possui pracinha/parquinho, situação parecida com a realidade examinada. No Brasil, a PeNSE em 2015, também levantou informações de estruturas para AF nas escolas e destacou a quadra de esportes disponível em 69,2\% das escolas das capitais do país, disponibilidade de vestiário em 22,2\% e pista de corrida e/ou atletismo em 1,6\%, para alunos da rede pública ${ }^{24}$.

No que diz respeito aos materiais encontrados, destacaram-se as bolas esportivas e materiais como colchonetes, cones, cordas e arcos. Esta informação pode estar relacionada à predominância de conteúdos de modalidades esportivas de quadra trabalhados nas aulas de Educação Física escolar. Em um estudo em Pelotas, com observação de 240 aulas de 16 escolas, os autores verificaram que modalidades esportivas (futsal, voleibol, basquetebol, handebol e futebol) foram os conteúdos mais trabalhados durante as aulas de Educação Física ${ }^{27}$. Em outro estudo, em seis cidades do Rio Grande do Sul, Pereira \& Silva ${ }^{28}$, em 2004, verificaram que quase 67,0\% das aulas de Educação Física foram destinados ao ensino de práticas esportivas, com conteúdo de ginásticas, coordenação motora e atividades lúdicas em menores percentuais de realização. Tal achado, também pode estar relacionado ao percentual de materiais de modalidades esportivas estarem disponíveis em con- dições regulares e ruins de uso, enquanto que cones, colchonetes, materiais de atletismo e ginástica apresentarem condições superiores de qualidade, indicando que os materiais de qualidade ruim podem estar mais desgastados devido ao seu uso frequente. Outro fator a considerar, tange sobre a autonomia de recursos das instituições para a compra de materiais, o que pode definir a variedade conforme a necessidade de sua realidade.

Em relação às atividades extracurriculares oferecidas, os torneios esportivos tiveram a maior prevalência, presentes em mais da metade das instituições. Esses dados são similares aos encontrados no estudo em Recife ${ }^{17}$, mas diferentes da zona urbana de Pelotas, onde as atividades extracurricualres predominates são dança e futsal ${ }^{18}$. Importante ressaltar que a diferença encontrada em relação às outras atividades pode estar relacionada à participação das escolas em campeonatos esportivos realizados pelos órgãos educacionais no município, como os Jogos Escolares de Pelotas e Jogos Escolares Rurais de Pelotas.

A ocorrência de lixo espalhado, mato e grama alta e animais soltos nas dependências do ambiente escolar, ressalta a importância de garantir melhores condições não somente estruturas físicas e materiais, mas que outros ambientes sejam convidativos e seguros para a sua utilização. Faria e Monlevade, em um documento do Ministério da Educação, tratam de temas de higiene e segurança na educação, cabendo à escola construir condições sanitárias adequadas e realizar manutenção de equipamentos e instalações, assim como garantir a segurança da comunidade escolar ${ }^{29}$. Embora a literatura nacional não tenha verificado questões sobre o tema, um estudo realizado na África do Sul mostrou que, mesmo que o espaço escolar esteja livre de objetos perigosos, resquícios de areia, cascalho e grama alta em áreas de lazer, estes espaços podem não estar propícios para promoção de estilos de vida saudáveis ${ }^{30}$.

Ao analisar o escore da qualidade das estruturas foi observado que escolas com maior número de alunos e com mais professores de Educação Física obtiveram maior média de pontos, bem como as escolas com ensino fundamental completo e com ensino fundamental e médio. Presume-se que essas características podem estar relacionadas à necessidade de as escolas estarem mais equipadas, estruturalmente e com maior quadro profissional, a fim de atenderem um maior número de alunos e com maior oferta das etapas de ensino.

Referente ao escore de qualidade dos materiais, escolas municipais, de ensino fundamental e com 150 a 299 
alunos obtiveram maior média. Acredita-se que as escolas de ensino fundamental, que geralmente estão sob a responsabilidade do governo municipal, podem possuir maior média no escore de qualidade dos materiais pelo fato de haver mais investimentos do poder público.

Alguns fatores limitantes do estudo devem ser considerados. A inexistência de um instrumento validado para mensurar o ambiente escolar na data da condução da pesquisa fez necessário a elaboração de um instrumento a partir de outros estudos. Entretanto, tal limitação não consiste em obstáculos para os achados da pesquisa. É importante destacar também a inexistência de estudos com escolas rurais. Isso pode ter dificultado a comparação dos achados do presente estudo.

A pesquisa teve como ponto forte se tratar de um censo, compreendo todas as escolas da zona rural do município de Pelotas. Ressalta-se também o fato de ter sido realizado na zona rural, região onde poucos estudos são desenvolvidos, destacando um ineditismo com o tema abordado.

O presente estudo buscou determinar, através da observação dos espaços escolares, a existência e condição das instalações e equipamentos para a prática de AF nas escolas da zona rural de Pelotas, Rio Grande do Sul. Pracinha/parquinho, quadras externas e campo de futebol, em condições ruins e regulares de preservação foram as instalações mais frequentes. Os equipamentos comumente encontrados foram cordas, cones e arcos, em boas condições de uso e bolas de voleibol, basquetebol, futebol, handebol e futsal, em pior estado de preservação. Torneio esportivo foi a atividade extracurricular mais promovida pelas escolas.

Os resultados mostraram que, apesar de a disciplina e do professor de Educação Física estarem presentes em todas as escolas, poucas instituições de ensino ofereciam instalações em condições adequadas para a prática de AF. A ausência de ginásios, quadras cobertas, espaços mais bem cuidados e materiais em boas condições de uso acenam para a necessidade de investimentos e intervenções nesses espaços.

Incentiva-se mais estudos sobre o ambiente escolar e, principalmente, a zona rural a fim de melhor compreender esses espaços e suas necessidades. São necessárias pesquisas que também explorem as ações da esfera política, organizacional e social dos ambientes escolares.

\section{Conflito de interesse}

Os autores declaram não haver conflito de interesses.

\section{Contribuição dos autores}

Müller WA, participou de todas as etapadas da pesquisa e escrita do artigo. Krüger GR, contribuiu com a redação do artigo e revisão crítica. Domingues $\mathrm{MR}$, orientou o planejamento e execução da pesquisa, colaborou com a análise dos dados, escrita e revisão final do artigo.

\section{Agradecimentos}

À Coordenação de Aperfeiçoamento de Pessoal de Nível Superior - CAPES, à Secretaria Municipal de Educação e Desportos de Pelotas e à 5a Coordenadoria Regional de Educação do Rio Grande do Sul.

\section{Referências}

1. Physical Activity Guidelines Advisory Committee. 2018 Physical Activity Guidelines Advisory Committee Scientific Report. Washington, DC: US Departament of Health and Human Services, ed. 2018: 779p.

2. Sallis JF, Bull, F, Guthold R, Heath GW, Inoue S, Kelly P, et al. Progress in physical activity over the Olympic quadrennium. Lancet. 2016;388(10051):1325-36.

3. Martins RC, Ricardo LIC, Mendonça G, Rosa DL, Bastos LLAG, Coll CVN et al. Temporal trends of physical activity and sedentary behavior simultaneity in brazilian students. J Phys Act Health. 2018;15(5):331-37.

4. Müller WA, Silva MC. Barreiras à prática de atividades físicas de adolescentes escolares da zona rural do sul do Rio Grande do Sul. Rev Bras Ati Fís Saúde. 2013;18(3):344-53.

5. Raphaelli CO, Azevedo MR, Hallal PC. Associação entre comportamentos de risco à saúde de pais e adolescentes em escolares de zona rural de um município do Sul do Brasil. Cad Saúde Pública. 2011;27:2429-40.

6. Bauman AE, Reis RS, Sallis JF, Wells JC, Loos RJF, Martin BW. Correlates of physical activity: why are some people physically active and others not? Lancet. 2012;380(9838):258-71.

7. Ferreira I, van der Horst K, Wendel-Vos W, Kremers S, van Lenthe FJ, Brug J. Environmental correlates of physical activity in youth - a review and update. Obes Rev. 2007;8(2):129-54.

8. Hoehner CM, Soares J, Parra Perez D, Ribeiro IC, Joshu CE, Pratt M, et al. Physical activity interventions in Latin America: a systematic review. Am J Prev Med. 2008;34(3):224-33.

9. Dauenhauer BD, Keating XD. The influence of physical education on physical activity levels of urban elementary students. Res QExer Sport. 2011;82(3):512-20.

10. Kremer MM, Reichert FF, Hallal PC. Intensidade e duração dos esfoços físicos em aulas de Educação Física. Rev Saúde Pública. 2012;46:320-6.

11. Haug E, Torsheim T, Sallis JF, Samdal O. The characteristics of the outdoor school environment associated with physical activity. Health Educ Res. 2010;25(2):248-56.

12. Kirby J, Levin KA, Inchley J. Associations between the school environment and adolescent girls' physical activity. Health Educ Res. 2012;27(1):101-14.

13. Melo EN, Barros M, Hardman C, Siqueira M, Wanderley Júnior R, Oliveira E. Associação entre o ambiente da escola de educação infantil e o nível de atividade física de crianças pré-escolares. Rev Bras Ati Fís Saúde. 2013;18(1):53-62. 
14. Knuth AG, Hallal PC. School environment and physical activity in children and adolescents: systematic review. Rev Bras Ati Fís Saúde. 2012;17(6):548-59.

15. Sallis JF, ConwayTL, Prochaska JJ, McKenzie TL, Marshall SJ, Brown M. The association of school environments with youth physical activity. Am J Public Health. 2001;91(4):618-20.

16. Morton KL, Atkin AJ, Coder k, Suhrcke M, van Sluijs EMF. The school environment and adolescent physical activity and sedentary behaviour: a mixed-studies systematic review. Obes Rev. 2015;17(2):142-58.

17. Tenório MCM, Tassitano RM, Lima MC. Conhecendo o ambiente escolar para as aulas de educação física: existe diferença entre as escolas? Rev Bras Ati Fís Saúde. 2012;17(4):307-13.

18. Knuth AG, Silva ICM, Hallal PC. Description of the school environment related to physical education classes, recess, extracurricular activities and physical spaces in the city of Pelotas, RS, Brazil. Rev Bras Ati Fís Saúde. 2015;20:524.

19. Brasil. Ministério da Educação. A Educação no Brasil Rural. In: Ministério da Educação/Instituto Nacional de Estudos e Pesquisas Educacionais Anísio Teixeira. ed. 2006: 236p.

20. Knuth AG. Ambiente escolar e prática de atividade física em crianças pertencentes à Coorte de Nascimentos de Pelotas de 2004 [tese de doutorado]. Pelotas: Universidade Federal de Pelotas; 2012.

21. Marques MO. Ambiente escolar e atividade física em escolares de Pelotas, RS [dissertação de mestrado]. Pelotas: Universidade Federal de Pelotas; 2012.

22. Lee R, Booth K, Reese-Smith J, Regan G, Howard H. The Physical Activity Resource Assessment (PARA) instrument: Evaluating features, amenities and incivilities of physical activity resources in urban neighborhoods. Int J Behav Nutr
Phys Act. 2005;2(1):1-9.

23. Silva AAP, Lopes AAS, Prado CV, Hino AFF, Reis RS. Características do ambiente físico e organizacional para a prática de atividade física nas escolas de Curitiba, Brasil. Rev Bras Ati Fís Saúde. 2018;23:10.

24. Brasil. Ministério da Saúde. Pesquisa Nacional de Saúde Escolar 2015. In: Ministério da Saúde/Instituto Brasileiro de Geografia e Estatística/Ministério do Planejamento, ed. 2015:132p.

25. Brasil. Lei no 9.394/96 de 20 de dezembro de 1996. Lei de Diretrizes e Bases da Educação Nacional. 1996

26. Silva MFP, Damásio MS. O ensino da Educação Física e o espaço físico em questão. Pensar Prát. 2008;11(2):6.

27. Fortes MO, Azevedo MR, Kremer MM. A Educação Física Escolar na cidade de Pelotas-RS: contexto das aulas e conteúdos. Rev Educ Fís. 2012;23(1):10.

28. Pereira FM, Silva AC. Sobre os conteúdos da Educação Física no Ensino Médio em diferentes redes educacioanis do Rio Grande do Sul. Rev Educ Fís. 2004;15(2):10.

29. Brasil. Ministério da Educação. Módulo 12: higiene, segurança e educação. In: Ministério da Educação/ Secretaria de Educação Básica, ed 2008: 75p.

30. de Villiers A, Steyn NP, Draper CE, Fourie JM, Barkhuizen G, Lombard CJ, et al. "HealthKick": formative assessment of the health environment in low-resource primary schools in the Western Cape Province of South Africa. BMC Public Health (online). 2012;12:1-11.

\section{Como citar este artigo:}

Müller WA, Krüger GR, Domingues MR. Características das instalaçôes e equipamentos para a prática de atividade física em escolas da zona rural de Pelotas, Rio Grande do Sul. Rev Bras Ati Fis Saúde. 2019;24:e0077. DOI: 10.12820/rbafs.24e0077 